\title{
Conceptual Localization of Yuri Stepanov's Semiotic Theory in Chinese Linguistics
}

\author{
Yu Zhang* \\ Siberian Federal University \\ 79 Svobodny, Krasnoyarsk, 660041, Russia
}

Received 28.09.2019, received in revised form 02.10.2019, accepted 10.10.2019

\begin{abstract}
In the article the author makes an attempt to localize the semiotic theory of Yuri Stepanov into the Chinese humanitarian scientific tradition, in which signs are considered as the main tool for the transmission of cultural concepts. Semiotics as an independent field became the subject of study of the Chinese linguists in the late 80ies of the last century, during the beginning of the policy of reform and openness. Chinese researchers in their papers refer to the significant works of outstanding representatives of the Russian semiotic school, namely, the works of M. Bakhtin, R. Jacobson and Y. Lotman. However, in the Chinese linguistics, according to the author, the theory of evolutionary-semiotic series of cultural concepts developed by Yuri Stepanov is insufficiently presented today. It has broad prospects of application to the Chinese semiotic space and interpretation of its cultural signs and codes. The present publication is intended to fill the existing gap in the research paradigm of the analysis of cultural layers in the structure of Chinese concepts.
\end{abstract}

Keywords: Chinese semiotics, Chinese cultural concepts, Yuri Stepanov, theory of evolutionary semiotic series of cultural concepts, semiotic portrait.

Research area: linguistic.

Citation: Yu Zhang (2019). Conceptual localization of Yuri Stepanov's semiotic theory in Chinese linguistics. J. Sib. Fed. Univ. Humanit. Soc. Sci., 12(10), 1965-1980. DOI: $10.17516 / 1997-1370-0499$.

(C) Siberian Federal University. All rights reserved

* Corresponding author E-mail address: Yuzhang0829@foxmail.com

ORCID: 0000-0002-4202-2895

This work is licensed under a Creative Commons Attribution-NonCommercial 4.0 International License (CC BY-NC 4.0). 


\section{尤里・斯捷潘诺夫的符号学概念阐释}

以及其在中国学界的本地化

西伯利亚联邦大学

文学与语言交际学院

东语教研室

俄罗斯，邮编：660041，克拉斯诺亚尔斯克，

自由大街79号

符号学作为一门独立的学科在上个世纪 80 年代就吸引了我国大量学者对其展开研究。然而 由于我国符号学起步较晚, 因此在符号学形成初期我国借鉴了大量外国学者的研究成果。 俄罗斯学界作为世界符号学发展的重要组成部分, 其符号学思想深深地影响着我国符号 学研究。其中我国学者更是对巴赫金、雅各布森、洛特曼等俄罗斯知名学者的研究成果进 行过深入地研究。然而经过分析, 本文作者发现我国学者对于俄罗斯后洛特曼时期以及俄 罗斯当代的符号学研究了解较浅。因此作者将通过本文向中国学者介绍、阐述俄罗斯后洛 特曼时期知名符号学学者尤里 - 斯捷潘诺夫的主要学术成果及学术概念。尤里・斯捷潘诺 夫作为当今俄罗斯学界的符号学杰出代表, 其推出的文化概念符号进化序列学说以及符号 肖像等概念至今仍被大量的俄罗斯学者使用在各个人文领域中。其研究成果具有庞大的潜 力, 而通过其推出的上述学说更是可以深入地分析位于文化深层的文化概念。本文旨在通过 对尤里・斯捷潘诺夫研究成果的阐述, 实现斯捷潘诺夫符号学思想在中国人文学术中的本 地化并使我国文化符号及文化概念研究能得到扩展。

关键词: 中国符号学、中国文化概念、尤里 - 谢尔盖耶维奇 - 斯捷潘诺夫、文化概念 符号进化序列学说、符号肖像.

研究领域: 语言学.

\section{1. 引言}

符号是人类在历史长河中保留至今没有遗失的重要宝藏。他们作为重要的交际 工具一直存在于人类社会当中并在人类的生活中扮演着至关重要的角色。作为一种 信息载体，符号不仅使人类的认知过程更加系统化、便捷化，还促进了交际的整体 过程，使交际过程当中的个人世界观、价值观的表达和呼唤更加精准。这在全球化 进程成为主流的今天更是意义非凡，不同国家、民族的个体正是通过使用各种不同 的符号体系从而实现自己国家、民族文化习俗、传统及价值观念的交流和互动。正如 我国著名学者祝东教授所说，使用符号传达意义是 “人类特有的功能”。而对于符 号具备意义传播作用这一现象的思考无论是我国还是西方国家都已存在已久。其中 较为著名的包括古希腊时期由希波克拉底提出的症候符号思想；亚里士多德提出的 语言逻辑符号思想；我国先秦时期哲学家所提出的易学中关于 “象” 的思考等。然而 由于上述思辨缺乏与之相应的理论及方法论因此普遍认为现代意义上的符号学诞生 于19世纪末20世纪初。其诞生学界一般归功于两位学者, 他们分别是美国著名哲学 
家、实用主义学家查尔斯・皮尔士和瑞士著名语言学家弗迪南・德・索绪尔。两人从未 有过任何学术上的交际和往来然而却几乎在同一时间提出了相同的关于符号科学的 概念。然而由于两人的学术领域不同，所以他们所提出的符号学概念也并相同，但 是两人对于符号学的形成及发展均起到了重要的作用。他们的著作也为其他各国学 者进行符号研究奠定了深厚的基础（Zhu Dong, 2014）。

符号学作为一门学科还很年轻, 然而在其短暂的发展历史中出现了很多优秀杰 出的学者, 例如法国的罗兰-巴特、俄罗斯的巴赫金、罗特曼等。伴随着符号学的发 展更多的学者加入到了符号研究当中。由于这些学者均来自不同的领域因此符号学 研究中形成了庞大的跨学科理论体系, 这也促使符号学研究形成了众多学派。苏俄 学派便是其中之一。俄罗斯作为现代符号学研究宗主国之一早在 19 世纪末 20 世纪初 便跟上了世界的学术潮流，开始了对于符号学的研究。20世纪的俄罗斯被当时的俄 罗斯政治哲学家尼古拉 - 亚历山德罗维奇 - 别尔佳耶夫称作为俄罗斯文艺复兴时期。 正是在这样的年代中, 由于新事物的流入, 学术研究逐渐从分散转向集中, 学者 们的注意也从个别学术领域转向了对于学术整体的研究和探讨。这一现象使学术研 究整体中的一些通用规则成为了学术研究的主要对象。符号研究在这样的大环境下 便自然而然的成为了当时俄罗斯学术界的主流研究之一。然而符号学作为一门独立 科学, 其在俄罗斯学术界的形成除了大环境的作用外还要归功于众多俄罗斯19世纪 中叶的著名学者, 其中包括韦谢洛夫斯基 (代表作: 《情节研究》、《历史史学中的三 章》)、克鲁舍夫斯基 (代表作:《语言学文章选集》)、波铁布尼亚（代表作:《思维与 语言》)、库尔德内 (代表作: 《普通语言学文选》) 等 (Pocheptsov, 1998)。作为19 世纪中叶的文学、语言学和语文学领域的代表他们对于符号学思想的传播和研究都 起到了重要的作用。虽然他们并没有提出系统化的理论体系, 也没有对符号学做出 详细明了的解说, 但是却为以后的符号学研究提供了较为科学的方法论及较为具体 详细的分析研究方法。而他们在各个文学、语言学及其他各个领域内的符号学初步 研究探讨更是为之后的符号学研究指明了方向 (Zhao Aiguo, 2008)。

进入20世纪后, 因在符号学研究上已有较为雄厚的基础, 所以俄罗斯的符号学 研究逐渐步入辉煌时期, 先后诞生了闻名世界的符号学学者, 例如: 梅耶尔霍尔德、 曼杰尔什塔姆 、雅库宾斯基 (代表作: 《关于对话性言语》)、什佩特（代表作:《现 象与含义》)、特鲁别茨科伊、雅各布森 (代表作: 《论俄语音系的演变》、《言语分析 初探》)、维诺格拉多夫 (代表作:《论文艺作品的语言》、《普希金的语言》)、巴赫金 (代表作:《陀思妥耶夫斯基诗学诸问题》、《小说中的词》)等 (Wang Mingyu, Chen Yong，2004)。他们作为20世纪的俄罗斯符号学代表完善了俄罗斯符号学已有的理论 体系并以此将符号学推上了新的高度。是他们将符号学研究带入了高潮阶段, 也正 是通过他们的研究, 符号学的研究方式方法逐渐被引入到很多其他的人文社会学科 当中。殷实的基础使俄罗斯的符号学发展迅猛。虽然在学科发展前期俄罗斯的众多 
学者均参考了索绪尔和皮尔士对于符号的思考和想法，然而后续的发展却离不开俄 罗斯本土学者的努力和研究 (Manaenkova，2013)。其中巴赫金和雅各布森的研究更 是漂洋过海被我国学者引入到了中国学界从而使我国符号学学者对符号学整体理论 的认知更加完善。引入国外名家理论的同时, 我国学者也在对这些理论进行研究, 甚 至是对一些理论思想提出疑问。其中我国著名符号学学者胡壮麟教授便对巴赫金的 符号学理论展开了深入的独立研究。1994年和2001年胡壮麟教授刊登了《巴赫金与 社会符号学》 以及《走进巴赫金的符号王国》两篇文章, 分别介绍了巴赫金的符号学 理论与思想并对其研究进行了系统化的分析 (Hu Zhuanglin, 1994, 2001)。2001年同年 我国另一位符号学学者蔡金亭教授也刊登了一篇与俄罗斯符号学理论相关的文章。 文章中蔡金婷教授对雅各布森提出的语言学标记理论进行了分析研究 (Cai Jinting, 2001)。除此之外对我国学界影响颇深的还有俄罗斯翻译领域的两位学者, 他们分别 是希罗瓦特金和巴尔胡达罗夫。前者提出了翻译是语码转换过程的概念; 而后者则 将符号学理论引入至翻译学当中。杨文全、方芳及周丽辉三位学者在自己的文章《现 代符号学理论对中国语言研究的影响》一文中便指出希罗瓦特金和巴尔胡达罗夫二 人的研究受到了我国学者的高度重视, 更是影响了我国翻译学领域的学者, 从而使 我国的翻译学研究也变得更加多样化 (Yang Wenquan, Fang Fang, Zhou Lihui, 2004)

正如我们上文所说的，由于俄罗斯学界中更多其他领域的学者参与到了符号学 研究当中，这也导致俄罗斯符号学学派众多。而其中最为著名的当属以俄罗斯知名 学者尤里 - 罗特曼为首的莫斯科-塔尔图学派该学派。该学派为现代俄罗斯符号学 的发展做出了杰出的贡献，而其学派代表尤里・罗特曼更可谓是划时代的符号学学 者。是他真正地将符号学带入了鼎盛时期。我国著名符号学学者王铭玉在其《语言符 号学》一书中更是用尤里 - 洛特曼来划分了俄罗斯符号学史的两个时代, 分别是洛特 曼时期和后洛特曼时期 (Wang Mingyu, 2004)。这样的划分并非无的放矢。由于20世 纪的俄罗斯符号学发展迅猛，当时的俄罗斯学术界诞生了众多的知名符号学学者。 其中尤里・罗特曼的众多研究更是将符号学的理论、学说、概念、研究方法带入了更 高的层次, 其著作更是具备着庞大的价值。在其逝世后俄罗斯的众多符号学学者也 一直在延续洛特曼的符号学研究, 从而导致今天的俄罗斯符号学逐渐地开始被上 世纪符号学研究的条条框框所限制 (Zhao Aiguo, 2008)。然而即便如此, 当今的俄 罗斯符号学仍不缺少惊艳的符号学学者。本文将重点介绍后洛特曼时期从众多学者 中脱颖而出的符号学学者尤里・希尔盖耶维奇・斯捷潘诺夫。

\section{1. 尤里・西尔盖耶维奇・斯捷潘诺夫}

20 世纪的俄罗斯符号学群星闪耀。众多的学派、学者, 各种各样的新的符号学理 论、概念、研究等层出不穷。然而正是在这样的年代中却有一位较为低调的学者, 他 
就是尤里 - 希尔盖耶维奇 - 斯捷潘诺夫。他是俄罗斯语言学基础理论的知名代表, 其 研究范围广泛, 无论是印欧语系语法还是逻辑语意学, 他均有涉及。此外他还是俄 罗斯知名的语言哲学家和理论家、著名翻译学者、文化学家、历史学家和宗教学者。 其在符号学领域内的贡献最为著名的便是其撰写的《符号学》 (《Семиотика») 一书以 及其收集翻译归类排版的《符号学著作选集》(《Семиотика/Антология》) 一书。前者 被誉为俄罗斯第一部也是最好的一部符号学书籍, 而后者则是俄罗斯学术界最为著 名的符号学选集之一。

相较于一位学者尤里 - 斯捷潘诺夫更像一位艺术家。这点从他对待学术的态度 就可以看出。尤里・斯捷潘诺夫一直认为学术同艺术一样源于生活, 学术与生活的联 系亦是紧密的。对于他来说科学认知并不是某种固定死板的群体知识生产和交换过 程, 而是一种活跃的、充满艺术性和美学的学者间的对话。这一点我们可以在尤里谢尔盖耶维奇与其他学者的对话中清楚地看到。每逢学术论坛或是其他活动时, 尤 里・谢尔盖耶维奇都仿佛一个听众一般, 他不会第一个参加到讨论中, 而是会去倾 听去理解， 甚至去捕捉演讲者或交际者还没有说出的下文，之后再开始对话。也正 是这样的特点使得尤里 - 谢尔盖耶维奇颇爱在自己的著作中使用引用的手法，并以 此完成自己与读者以及所研究学术领域先代学者的对话。在其于1985年发表的《语言 的三维空间》 (《В трёхмерном пространстве языка»)一书的引言中, 尤里・谢尔盖 耶维奇曾这样说过: “这本书中有很多引言。我们曾想让我们的著作符合传统的方法 论, 将更多的学术历史文化发展过程中参与人的声音带入到著作当中, 其中当然也包 括思想家和一些学术文章的作家。但是总是有人会问 “作者的声音呢?”这样的问 题就像要求声乐合唱指挥唱歌一样毫无意义, 因为某种程度上来说作者和合唱乐队 指挥的作用是一样的。” (Stepanov, 1998, p. 179)。正是这样的特点使尤里・斯捷潘 诺夫成为了一位优秀的学者。他相较于其他学者, 集百家所长, 研究范围广泛。而在 其研究中, 尤里-谢尔盖耶维奇更是侧重于语言在多个领域内的运用方式, 其中包 括了科研学术、艺术、哲学、宗教、神话学等。这也致使其科研成果具备跨专业和多 元化的特性 (Postovalova, 2012: 48-63) 。

\section{2. 尤利・谢尔盖耶维奇・斯捷潘诺夫研究中的符号学}

尤里・谢尔盖耶维奇的研究中, 作者对于符号学的定义较为广泛。他认为符号学 是一门研究社会和自然中存在的符号体系的学科 (Stepanov, 1998, 19) 。而符号学 的研究对象也一直是尤里 - 斯捷潘诺夫学术生涯中的创作源泉。在自己的专著《符号 学》 (《Семиотика»)一书的序言中, 它更是精准地指出符号学的研究对象实际上就 是信息系统 (информационная система): “符号学的研究对象很广泛, 其中包括 了语言、数学、文学作品、建筑、房间装潢、家庭的建设、人类的潜意识活动、动物间 的交际、生物的生长等。但是他们都具备一个共同点,

那就是他们都是作为信息载 
体和信息系统而存在。而他们的核心便是我们所说的符号系统了” (Stepanov, 2001, p.5)。

此外, 尤里 - 斯捷潘诺夫还曾提出符号学具备更加庞大的跨学科潜力这一假说。 在分析当时已存在的符号学分支（也就是生物符号学、民族符号学、文化符号学以 及语言符号学）时，尤里・斯捷潘诺夫便指出符号学研究已经开始涉及心理学和认 知学方面的领域, 而心理分析更是开始成为符号学的研究对象。而后, 他更是提出 符号学研究范围将更加广泛，更加专业化。在2001年出版的专著《符号学世界》(《B мире семиотики») 一书当中, 尤里・斯捷潘诺夫更是提出符号学研究已经不局限 于现有的符号学领域研究了, 它已经开始向其他领域扩展, 其中之一便是伦理学。 这一领域内以符号学为基础已经展开了众多的研究，其中较为著名的由道德假设的 符号性质 (семиотический статус морального постулата)。针对该领域内的符号 研究斯捷潘诺夫先生依据皮埃尔・泰亚尔・德・夏尔丹关于人的假说指出这类研究 应该 “侧重于人的人格而非单独的个体” (стремиться не к индивидуальности, а к личности)。另一个引入符号学理论和研究方法的领域为文化学。尤里・斯捷潘诺 夫谈到文化学时指出从符号学的角度出发分析研究文化概念也将成为一种主要的符 号学研究潮流 (Stepanov，2001：40)。文化学内的符号研究也成为了尤里・斯捷潘诺 夫学术生涯中的重点研究。此外尤里・斯捷潘诺夫和大多数符号学家一样在自己的 研究中探讨过语言与符号学的关联。在创作《语言与方法》 (《Я з ы к и М е тод») 一书的过程中, 尤里 - 斯捷潘诺夫总结自己在语言哲学领域内的研究结果时曾这样 说过： “对于我这个作者来说，最让我意想不到的便是构成这本书的研究成果。他 们均为我在不同的年代进行的研究，但是他们的发展，本质上遵从了与符号学发 展相符的发展进程” (Stepanov, 1998: 12)。从他的研究中也确实可以发现, 斯捷 潘诺夫在语言哲学领域内大部分的研究均是根据二元一体 “能指” 与 “所指” («Означаемое / Означающее»); 以及三元一体 “句法”、“语义”与 “语用” («Семантика / Синтактика / Прагматика»); 和 “体系”、“文本” 和 “话语” («Система / Текст / Дискурс») 为基础而进行的 (Postovalova, 2012: 3)。

在《符号学世界》(《B мире семиотики») 一书当中, 尤里・斯捷潘诺夫 还提出了一种新的符号学概念。他提议将符号束和符号系统作为一种信息空间 (информационное пространство) 来看待。这样的概念促使他在后续的符号学研 究中曾把符号学定义的非常广泛，以至于作者的观念中符号学开始逐渐等同于人 类学 (антропология)。这使得尤里・谢尔盖耶维奇开始将符号学与认知学联系在一 起 (Stepanov，2001：31)。早在1998年时作者就开始了这类研究。其最初对于符号 学的定义便是通过符号学角度研究认知学的一种经验 (опыт введения в теорию познания от семиотики) (Stepanov, 1998: 35-36)。这样的研究方向一直持续了多年 直至尤里 - 斯捷潘诺夫开始分析研究上世纪 50 年代的语用学。在研究过程中他发现 
符号的因素之间缺少一种自然关系。符号与其所代表的物体之间也同样缺少这种自 然关系。这样的发现使尤里 -谢尔盖耶维奇停止了其结合符号学与认知学的研究方 法, 转而开始开创一种独特的结合唯名论与实在论的符号学科研方法 (некоторого синтеза реалистических и номиналистических идей) (Stepanov, 1998: 612)。

尤里・斯捷潘诺夫的研究中我们可以看到其研究对象普遍为符号学作为一种学 科的一些具体现象, 例如符号学的起源、发展, 符号学研究的基础等等。然而在其 学术生涯中其作为重点研究对象的当属符号与其所代表的物体之间的关系。他曾提 出多个对于俄罗斯当今学界仍然现实的问题, 其中就包括了这样的一个问题： “代 表某种精神文化概念(концепты духовной культуры)的符号能否完全阐述表达其所 代表的概念呢? ” 在回答自己提出的这个问题的时候，尤里 - 斯捷潘诺夫也曾自己 说过：“我们对精神文化概念的阐述是有限的。例如对于 “信仰”、“爱情”、“真 理” (концепты «Вера», «Любовь», «Истина») 这样的概念, 我们只能局部地阐述 他们，因为在这些概念的背后是某种文化现实，我们无法用语言表达、阐述、描写， 只能用心去感受。” 无里・谢尔盖耶维奇后续关于精神文化概念符号的研究一直以 概念 “信仰” (концепт «Вера») 为主进行。在自己的文章中作者曾指出 “信仰” 这 种精神文化概念 (концепт духовной культуры) 不仅可以代表一种现象，还可以 表达不同个体的内在精神状态。而关于怎样描写这种个体的内在心理状态，尤里・ 斯捷潘诺夫曾指出 “纵使当今科学发达, 但是描写每个个体的不同内在心里状态已 经是科学认知以及概念分析描写的极限了。” (Stepanov, 2001: 76)

尤里・谢尔盖耶维奇关于概念的研究还促使了另一门符号学分支的诞生 - 宗教 符号学 (религиозная семиотика)。而用以支撑这门分支的恰恰是尤里・谢尔盖耶维 奇关于新现实主义的学说。他的学说中除了现实主义哲学以及一些艺术美学概念外, 还包括了东方基督教父学的理论及学说。这些学说中非常详细地从符号学的角度分 析解读了基督教的圣象、教堂等。后续这类研究更是成为了俄罗斯学界较为独特的 一种符号学分支 (Postovalova, 2012: 52)。尤里 - 斯捷潘诺夫本身也从符号学的角度 对宗教学进行过研究， 其在宗教符号学的框架下提出的 “神人” 的概念也为宗教符 号学的发展起到了重要的作用。

3. 尤利・谢尔盖耶维奇 - 斯捷潘诺夫符号学研究角度下的文化与概念

正如我们上文中所说的一样, 尤里-谢尔盖耶维奇的符号学学派主要是从符号 学研究的角度去分析研究认知学中的概念(концепт)。作为学派的创始人, 尤里 - 斯 捷潘诺夫在研究概念的过程中也逐渐开始涉及与文化相关的概念，甚至提出了一 种文化概念的符号进化序列学说 (Теория эволюционно-семиотических рядов концептов культуры) (Sudakova, 2017: 61)。 
文化概念符号序列进化学说 (Теория эволюционно-семиотических рядов концептов культуры) 源于作者于90年代初期结合认知学、符号学与语言学的研 究。这些研究当中尤里 - 斯捷潘诺夫主要提出应从符号学的角度去研究文化与概念 两者间的联系。研究结果以学术文章的方式于1991年、1992年刊登并收录在俄罗斯学 术杂志《语言逻辑分析》 (《Логический анализ языка») 当中。其中尤为重要的为一 篇名为《概念 “理由” 以及两种语言概念分析法：逻辑法与子逻辑法》 (《Концепт “причина" и два подхода к концептуальному анализу языка - логический и сублогический») 的文章。这篇文章中尤里・斯捷潘诺夫首次指出当个体将概 念融入到语言当中, 概念就会具备文化意义与认知意义(культурно-когнитивный смысл) (Stepanov, 1991)。而在1992年与另一位俄罗斯知名符号学学者普罗斯库林合 作刊登的《世界文化中的 “行动” 概念》 (《Концепт “действия” в контексте мировой культуры») 一文中尤里 - 斯捷潘诺夫由提出了自己对于文化的推论。他认为文化是 一个符号集体(семиотическая целостность)，在这个集体中代表物体层面的概念 (материальные ряды)以及代表精神层面的概念(ряды духовных концептов)并列存 在。他们会与代表自己的符号（此处的符号特指语言符号）产生联系, 随后被个体 分类成不同的种类 (Stepanov, Proskurin, 1992)。此外在这篇文章中两位作者还谈及 了概念的形成过程。他们指出概念是以抽象的方式(путем абстракций) 通过对文化 的物质和精神层面探索从而同时或根据一定的顺序形成的一种认知结果。也就是说 新概念的形成是通过个体对世界的探索而形成的。而之后这个形成的概念又会成为 形成某种新概念的基础并如此反复。这使概念具备了两种分类，一种是属于某个文 化精神层次的概念; 另一种则是即属于精神层次也属于物质层次的概念。这使新概 念形成的同时，会产生一种新旧概念的融合，从而使新的概念在保留原概念局部含 义的同时形成属于其独特的符号层级 (семиотические слои)。这一现象证实了尤里斯捷潘诺夫关于文化概念具备复杂构造的假设 [同上]。

在讨论概念的发展进化方式时，无利・斯捷潘诺夫曾指出概念的发展方式与爱 德华・泰勒提出的文化进化论非常相似。但是相较于泰勒的文化进化论, 尤里・斯 捷潘诺夫提出的文化概念符号序列进化学说指出，不同的毫无关联的概念可以互相 交叉从而在保留两种概念内在含义的同时形成某种新的概念，并以此为基础层层叠 加发展成其他的概念。原概念内在含义的排斥会在新概念形成初期产生, 但随后原 概念的内在会流入新概念的符号层面当中 [同上]。

尤里 - 斯捷潘诺夫关于文化概念的完整研究出版于1997年。这本名为《常量: 俄罗斯文化词典：研究报告》 («Константы: словарь русской культуры: опыт исследования») 的专著受到了当时俄罗斯学界的广泛认可。在这本著作中尤里・斯 捷潘诺夫首次提出了自己对于文化整体的探讨。由于尤里・斯捷潘诺夫本身对于学术 研究的严谨态度, 因此当他将文化定为研究课题时他便指出不应以自己的研究角度 
和主观看法去定义自己研究中的文化。他借鉴了英国著名民族学者、社会学家布罗尼 斯拉夫 -马林诺夫斯基与俄罗斯知名社会学家皮特林・索罗金两位学者关于文化的 研究并以此证实了自己关于构成文化内在体系的因素是互相紧密相连的假设。随后 尤里斯捷潘诺夫更是在此基础上提出了自己对于文化的定义。他认为文化是某种空 间 (некое пространство)。在这片空间中所有已经存在的词汇、思想、概念都能与 新的词汇、思想和概念产生联系，他们相互沟通形成反馈并以此形成一种特殊的和 谐共鸣(гормоничное созвучание)。正是这种共鸣将这些词汇、概念和思想归类成 不同的进化符号序列 (эволюционно-семиотические ряды)。这种以序列进化原则 (эволюционно-рядовой принцип) 为基础的系统化归类方式更接近于文化的物质 层面, 然而尤里・斯捷潘诺夫则认为这样的方式同样适用于归类文化精神层面的现 象和概念。其原因便是任何一个文化现象都具备的两面性特质。这种两面性主要体 现在现象的物质层面也就是现象所代表的某个物体, 亦能体现在现象的精神层面中 代表这个物体的精神符号。此外在文化概念进化符号序列学说中, 符号学本身的作 用是不可忽视的。正是通过符号学尤里・斯捷潘诺夫才能将一个个看似毫不相干的 文化概念联系在一起并按照进化顺序将他们排列成一种符号序列。在这个序列中, 所有的概念都具备着 “承上启下” 的作用，他们在继承原概念内在含义的同时也会 为新概念的形成奠定一定的基础（Stepanov，2001：14）。尤里・斯捷潘诺夫在该领 域的后续研究中还以上述学说为基础推出了文化概念符号序列同步 (синхронные семиотические ряды культуры)。学者将时间作为考量因素融入到了文化概念符号 序列进化学说当中。这里所指的时间并非某个时间段, 而是一个特定的时间点。在这 个特点的时间点代表某种现象或事物且分属不同概念符号序列的文化概念，会在某 个文化所覆盖的多个地区内或某个国家的不同地区内同时发生、产生联系并形成思 维等语线序列 (ряды ментальных изглоссов) (Stepanov, 2001: 39, 41)。

在该学说的范围内, 尤里 - 斯捷潘诺夫还提出了文化学中的概念内在构成 (внутренне строение концепта)。他指出由于文化中的概念存在于不同的文化层 级, 这导致有的概念（例如一些专业术语中的概念）只被少数人掌握并使用, 而 有的概念则恰恰相反（例如一些日常用语中的概念）他们被不同社会阶级的人 广泛使用。这也导致文化中的概念具备了三种不同的层级, 他们分别是：实际含 义 (актуальное содержание)、被动含义(пассивное содержание) 以及原本含义 (буквальное содержание)。概念的不同层级也具备着不同的作用。其中概念实际 含义层级的主要作用便是将概念作为一种沟通和互相理解的工具将所有使用某种 语言的个体团结在一起。而被动含义层级则在将个体团结在一起的同时将他们按 照社会层级进行分类。原本含义层级或原始含义层级则较为特殊。该层级一般出现 在学者们的调查当中。他们会通过对话语中包含的文化现象 (явления культуры, которые заключены в словах) 进行分析以此确定某个概念的原始含义, 所以原始 
含义层级也被称为学术含义层级 (научный слой)。换句话说, 尤里・斯捷潘诺夫的 研究中概念不仅是作为一种个体认识工具存在, 还被视为一种社会群体文化传承 (коллективное наследство культуры) (Sudakova, 2017: 64)。

综上所述，尤里・斯捷潘诺夫关于文化领域的研究与概念和符号都具有非常紧 密的联系。他认为概念不仅是个体用来认识了解世界的工具, 更是一种个体用来了 解所在文化的工具。由于概念在该领域内被尤里 - 斯捷潘诺夫定义为社会群体文化 的传承方式和传承结果，所以概念可以表达很多位于文化精神层面的现象，而概 念恰恰是以符号的形式在现实世界中具现化。因此大到民族文化，小到某个社会群 体、集团或家族的文化我们都可以使用概念和符号去沟通描写他们，并通过与之相 对应的文化概念符号序列来了解某个文化在不同时期的主要现象以及他们是如何通 过概念和符号将这些现象具现化并传承至今。这使此类的研究相较于常规的文化历 史分析或社会分析要更加广泛。

\section{4. 尤利・谢尔盖耶维奇・}

斯捷潘诺夫符号学研究角度下的宗教信仰以及关于个体与人的探讨

通过前文的描写我们不难看出尤里・斯捷潘诺夫的研究中，符号与概念的联系 是紧密的。他们作为表达和描写文化的两个重要要素一直被尤里・斯捷潘诺夫运用 在文化符号学研究当中。关于符号的概念表达性质斯尤里 - 捷潘诺夫曾在他出版的《 符号学》(《Семиотика») 一书中指出 “符号具有不同层级的表达性质。正是这些性 质造就了符号对于概念的表达能力，换言之符号的意义值是不同的，是波动的”。之 后在1998年再版的《语言与方法》(《Язык и Метод》) 一书中尤里・斯捷潘诺夫又根 据符号的表达性质对他们进行了分类，他们分别是：等同于所表达的概念或物体的 符号(знаки, идентичные с объектом); 与所表达的概念或物体部分相同的符号, 即 符号不能完全表达描写的概念或物体 (знаки, подобные объекту); 以及在特定的 环境和条件下根据使用符号的个体从而与某个概念或现象产生联系的符号 (знаки, условные/конвенциональные по отношению субъекту)。这与哲学和美学中的符 号分类非常相似 (Postovalova，2012）。

在对俄罗斯文化进行文化符号学研究时，由于俄罗斯文化中宗教信仰作为一种 核心概念存在，因此尤里・斯捷潘诺夫开始围绕宗教进行符号学研究时他便陷入了 一种困境当中。因为宗教信仰是一种较为抽象的概念，所以学者发现在描写该概念 时语言符号的表达已经不完整了, 或者说概念 “信仰” (концепт “Вера”) 的意义 值已经超出了语言符号的表达范围。单单使用由语言符号构成的语料已经不足以完 全阐明俄罗斯文化的核心概念 “信仰”。于是学者逐渐将自己的目光放到了同样为 符号一种的象征符号 (символ) 上。尤里 - 斯捷潘诺夫对于象征符号的探讨同样出现 在《语言与方法》 (《Язык и Метод》) 一书当中。书中学者将象征符号视为一种艺术 
的最高级别的表现手法, 正是通过象征符号才能完整地表达出艺术和文化的精神层 面 (Stepanov, 1998: 234)。

讨论到俄罗斯文化中的核心概念 “宗教信仰” 时，尤里・斯捷潘诺夫提到了俄 罗斯宗教中的 “神人” 或 “半神半人” 概念(концепт “Богочеловек”)。在自己以 希腊海神命名的的著作《普罗透斯》(《Протей》) 一书中，尤里・斯捷潘诺夫便开始 以欧洲国家和文化宗教中半神（这里特指耶稣）的形象为基础进行自己的研究。为 了保证研究的完整, 学者不仅研究了描写耶稣的语料, 还研究了作为象征符号一种 的图像, 也就是画像和一些圣象。而其研究使用的主要研究材料为俄罗斯十九世纪 著名作家费奥多尔・米哈伊洛维奇・陀思妥耶夫斯基以及荷兰十七世纪著名画家伦勃 朗・哈尔曼松・范・莱茵的作品。研究的过程当中，学者指出虽然两位创作家分数不 同的艺术领域，但是他们笔下的耶稣都具备一个共同点，那是一种完美的人类形象 (образ идеального человека)。同时两位创作家还将耶稣的完美归于他灵魂中的神 性，换言之他们的作品中展示出的不仅是耶稣，更是人性与神性的结合 (Stepanov, 2004)。

上述研究成果最后被尤里 - 斯捷潘诺夫整理并收录在自己的专著《普罗透斯》 (《Протей») 一书中。其中在这本书的终章《当代半神形象》 («Богачеловек в наши дни») 时，尤里・斯捷潘诺夫还曾对于 “半神” 的概念提出了一些探讨。他提出了这 样的一个问题 “半神这个名称背后隐藏的究竟是一种概念还是一种形象?” 而随 后他便在后面的文章中自己给出了这个问题的答案。他认为只有在概念和形象结合 时，才能更为准确的定义文化概念 “半神” 的含义。这也是为什么学者在研究时 才使用了陀思妥耶夫斯基以及伦勃朗的作品（同上）。

随后的研究中尤里・斯捷潘诺夫更是提出了一种新的术语 “形象概念” (oбpa3концепт)。学者提出的形象概念与俄罗斯著名哲学家、文化学家阿维林策夫 - 谢尔 盖・谢尔盖耶维奇关于象征 (символ) 的探讨非常相似。阿维林策夫认为象征是一 种出自某种含义、概念并用于表达他们的形象。这种形象可以被定义为某种包含有限 信息但形态丰富的符号。象征的结构中，其外在的形态以及其内在的深刻含义形成 了象征的两极, 他们是分散的但是同时也是缺一不可的。而他们之间的联系恰恰就是 象征的本质（Averintsev，2001）。这与尤里・斯捷潘诺夫的形象概念不谋而合，只 有通过符号的内在和外在形态的结合才能抵达符号和概念更深的层面。

回到对于概念 “半神” 的探讨, 区别于其他传统的宗教哲学家，尤里 - 斯捷潘诺 夫作为一个符号学家, 在研究宗教和信仰时最令他感兴趣的莫过于耶稣的本质了。 通过对陀思妥耶夫斯基的文学作品以及伦勃朗画作的分析, 学者在自己的研究中认 为耶稣在作为一个神明之前是一个真实的、完美无缺的人。这时尤里 - 斯捷潘诺夫在 原有的形象概念基础上提出了著名的符号肖像 (семиотический портрет) 概念。他 认为必须通过符号肖像的方式才能论证耶稣作为神明的人性。 
对于肖像的定义，尤里・斯捷潘诺夫的描写较为特殊。他从以下三方面看待符号 肖像:

1）认识论方面 (в плане гносеологии): 在这个角度下，尤里・斯捷潘诺夫对于 肖像的定义较为模糊。他以个体思维中的内在世界为例, 指出认识论中的肖像仿佛每 个个体思维中的世界一样, 是一种符合逻辑和常识但不一定真实存在介于现实与幻 想之间的一种映像(Stepanov, 1998: 234)。

2) 话语方面 (в плане дискурса): 关于这方面的探讨主要收录在尤里・ 斯捷潘诺夫《思考的芦苇：关于 “幻想文学”》(Мыслящий тростник. Книга о “Воображаемая словесность”) 一书中。其中学者认为肖像是一些景色和人物的 图像, 它们不仅作为一种视觉成像存在, 有时更是话语一部分甚至是话语的来源 (Stepanov, 2010)。

3）符号学方面 (как семиотический фактор)：谈及符号肖像时，尤里・斯捷潘 诺夫曾这样评价过: “和肖像相比, 没有任何一个概念在进入符号学时会变得如此 复杂”。在《普罗透斯》一书中，尤里・斯捷潘诺夫关于符号肖像的大部分探讨都源 于哲学以及一些基督教的宗教思想。他认为符号学角度下的肖像代表的是 “人的本 质” (“сущность человека”)、“个体的本质” (“индивид”)。尤里・斯捷潘诺夫还特 别说明, 这里的本质指的并不是人的外在(外表、生理形态等), 也并非是心理学和 社会学中所说的个体, 而是宗教哲学家所研究的三位一体和位格 (ипостась), 换言 之便是人性中蕴含的神性 (божественная сущность) (Stepanov, 2004)。

通过符号肖像尤里・斯捷潘诺夫逆向推论论争了耶稣的人性，同时也论争了困 扰自己多年的议题: 逻辑概念或真理是在什么情况下通过什么方式才会具备个人色 彩? 经过对于符号肖像的研究学者发现正是在符号肖像中永恒的真理才会成为一种 个体的逻辑真理。他在《普罗透斯》的终章中指出个体的认知过程中接触认识真理是 一个必要的过程。而在接触认识真理时个体又会将他们自动归类为神的真理，但是 由于个体内在的 “神性” 真理也会逐渐具备个人色彩。换言之，每个个体即主观阐 述个人逻辑真理也阐述客观真理。用著名德国哲学家和逻辑学家戈特洛布・弗雷格 （F. Gottlob）的话来说个体在阐述观念时根据自己的日常形象将自己内在的神性形 象融入到自己所阐述的真理或事实当中（同上）。

\section{5. 结语}

尤里・斯捷潘诺夫作为连接两个时代的符号学学者, 其涉足的研究领域远远超 过我们文章中所列举的那些。虽然没有较高的知名度, 但是他对俄罗斯符号学的发 展做出了非常大的贡献。尤里・斯捷潘诺夫创作的诸多研究中令人印象最深刻的便是 其研究之间的连贯性了。学者大部分的文章之间都存在着柔和的承上启下关系。此 外最令人难忘的当属学者对于学术研究的看法与观念。尤里 - 斯捷潘诺夫不愧为学 
术界的艺术家，他对于学术理论勇敢大胆的创新为俄罗斯后续的符号学发展提供 了巨大的动力。其提出的文化概念符号序列进化学说以及形象概念和符号肖像等概 念具备着非常大的学术能量。至今仍被俄罗斯学者广泛运用在很多人文领域研究当 中并不断地被继承、传承着。

通过这篇文章我们介绍阐述了尤里・斯捷潘诺夫在符号学中的主要研究成果。 其研究成果具备丰富的理论和实践价值。这里我们重点要提及的是一些可以引用尤 里-斯捷潘诺夫研究的课题。正如我们全文所说的一样, 尤里・斯捷潘诺夫的主要研 究方向为符号学角度下的文化。所以其研究成果用于我国文化以及我国神话传说的 研究当中是可行的。相信通过学者提出的文化概念符号序列进化学说我们会更加完 整、完善地认识祖国文化以及文化中蕴含的一些深层次的文化概念。而通过符号肖像 则可以对我国神话传说中的人物进行更加深层次的分析，从而形成更为精准的人物 形象并探明人物更为深层面的特质和人物的本质。此外关于符号肖像的研究同样可 以用于文学人物分析当中, 以获得更加鲜明的角色形象。

\section{References}

Averintses, S.S. (2001). Sofiia - Logos: Slovar' [Sofia - Logos: Dictionary]. Kiev, Dukh i Litera, $450 \mathrm{p}$.

Cai Jinting (2001). Yagebusen de yuyan biaoji lilun: chengjiu yu buzu [Jakobson's Language Marking Theory: Achievements and Deficiencies]. In Waiyu xuekan [Foreign language journal], 2, 78-84.

Hu Zhuanglin (1994). Bahejin yu shehui fuhaoxue [Bakhtin and Social Semiotics]. In Beijing daxue xuebao [Journal of Peking University], 2, 49-56;

$\mathrm{Hu}$ Zhuanglin (2001). Zoujin bahejin de fuhaoxue wangguo [Entering Bakhtin's semiotic kingdom]. In Waiyu yanjiu [Foreign language research], 2, 10-15.

Manaenkova, E.A. (2013). Semioticheskie shkoly i napravleniia [Semiotics Schools and Directions]. In Analitika kul'turologii [Cultural Studies Analytics], 27, available at: https://cyberleninka.ru/article/n/semioticheskie-shkoly-i-napravleniya

Pocheptsov, G.G. (1998). Istoriia russkoi semiotiki do i posle 1917 goda: Uchebnospravochnoe izdanie [The History of Russian Semiotics Before and After 1917: Educational-Reference Publication]. Moscow, Labirint, 336 p.

Postovalova, V.I. (2012). Simvol i real'nost' (semioticheskie vozzreniia i opyty Iu. S. Stepanova) [Symbol and Reality (Semiotic Views and Experiments of Yu. S. Stepanov)]. In Kritika i semiotika [Criticism and Semiotics], 17, 123-142.

Stepanov, Iu.S. (1991). Kontsept «prichina» i dva podkhoda k kont septual'nomu analizu iazyka - logicheskii i sublogicheskii [The Concept of "Cause" and Two 
Approaches to The Conceptual Analysis of Language - Logical and Sublogical]. In Logicheskii analiz iazyka: kul'turnye kontsepty [Logical Analysis of Language: Cultural Concepts], 5-14.

Stepanov, Iu.S. (1997). Konstanty: slovar' russkoi kul'tury. Opyt issledovaniia [Constants: a Dictionary of Russian Culture. Research Experience]. Moscow, Iazyki slavianskoi kul'tury, $825 \mathrm{p}$.

Stepanov, Iu.S. (1998). Iazyk i Metod: $k$ sovremennoi filosofii iazyka [Language and Method: Towards the Modern Philosophy of Language]. Moscow, Iazyki russkoi kul'tury, $784 \mathrm{p}$.

Stepanov, Iu.S. (2001). Vvodnaia stat'ia. V mire semiotiki [Introductory Article. In the World of Semiotics]. In Semiotika: Antologiia [Semiotics: Anthology], 5-42.

Stepanov, Iu.S. (2004). Protei: Ocherki khaoticheskoi evoliutsii [Proteus: Essays on Chaotic Evolution]. Moscow, Iazyki slavianskikh kul'tur, 272 p.

Stepanov, Iu.S. (2010). Mysliashchii trostnik. Kniga o «Voobrazhaemol slovesnosti» [Thinking Reed. The Book About "Imaginary literature"]. Kaluga, Eidos, 169 p.

Stepanov, Iu.S., Proskurin, S.G. (1992). Kontsept «deistvie» v kontekste mirovoi kul'tury [The Concept of "Action" in The Context of World Culture]. In Logicheskii analiz iazyka: modeli deistviia [Logical Analysis of the Language: Models of Action], 5-14.

Sudakova, O.N. (2017). Semioticheskaia kontseptualizatsiia kul'tury v rabotakh Iu. S. Stepanova [The Semiotic Conceptualization of Culture in The Works of Yu. S. Stepanov]. In vestnik Sankt-Peterburskogo gosudarstvennogo instituta kul'tury [Bulletin of The St. Petersburg State Institute of Culture], 2(31), 61-64.

Wang Mingyu (2004). Yuyan fuhaoxue [Linguistic Semiotics]. Beijing, Gaodeng jiaoyu chubanshe, $563 \mathrm{p}$.

Wang Mingyu, Chen Yong (2004). Eluosi fuhao yanjiu de lishi liubian [Historical Evolution of Russian Semiotic Studies]. In Dangdai yuyanxue [Contemporary linguistics], 6 (2), 159-168.

Yang Wenquan, Fang Fang, Zhou Lihui (2004). Xiandai fuhaoxue lilun dui zhongguo yuyan yanjiu de yingxiang [The Influence of Modern Semiotics on Chinese Language Studies]. In Qinghai minzu xиeyuan xuebao (shehui kexue ban) [Journal of Qinghai Nationalities University (Social Science Edition)], 4, 152-156.

Zhao Aiguo (2008). Ershi shiji eluosi fuhaoxue yanjiu de lishi fenqi wenti [The Historical Staging Problem of Russian Semiotic Studies in the $20^{\text {th }}$ Century]. In Jiefangjun waiguoyu xueyuan xuebao [Journal of PLA University of Foreign Languages], 4, 31-35. 
Zhu Dong (2014). Xianqin fuhaoxue sixiang yanjiu [Research on The Semiotic Thoughts of The Pre-Qin Period in China]. Chengdu, Sichuan daxue chubanshe, 246 p.

\title{
Концептуальная локализация понятий семиотической теории Ю. Степанова \\ в китайском языкознании
}

Чжан Юй

Сибирский федеральнылй университет Россия, 660041, Красноярск, пр. Свободный, 79

\begin{abstract}
В данной статье автор осуществляет попытку локализации в китайскую гуманитарную научную традицию семиотической теории Ю.С. Степанова, в которой ученый рассматривает знаки как основной инструмент передачи культурных концептов. Семиотика как самостоятельная область стала предметом изучения китайских лингвистов в конце 80-х годов прошлого столетия, в период начала политики реформ и открытости. Китайские исследователи в своих изысканиях обрашаются к значимым трудам ярких представителей российской семиотической школь, а именно работам М. М. Бахтина, Р. О. Якобсона и Ю. М. Лотмана. Однако в китайском языкознании, по мнению автора, на сегодняшний день недостаточно представлена теория эволючионно-семиотических рядов кониептов культуры, разработанная Ю. С. Степановым, которая имеет широкие перспективы приложения к китайскому семиотическому пространству и дешифрованию его культурных знаков и кодов. Предлагаемая публикация призвана заполнить существующую лакуну в исследовательской парадигме анализа культурных наслоений в структуре китайских концептов.

Ключевые слова: китайская семиотика, китайские культурные концепты, Юрий Сергеевич Степанов, теория эволючионно-семиотических рядов концептов культуры, семиотический портрет.
\end{abstract}

Научная специиальность: 10.02.00 - языкознание. 